\title{
COSMOLOGY WITH GALAXY CLUSTERS THROUGH THE SUNYAEV-ZEL'DOVICH EFFECT
}

\author{
J.M Diego \\ Physics Dept. Denys Wilkinson Bldg, Keble Road, Oxford OX1 3RH, U.K. \\ jmdr@astro.ox.ac.uk
}

\begin{abstract}
Future Sunyaev-Zel'dovich effect (SZE) surveys will detect thousands of clusters, many of them at high redshift. We show how the SZE number counts as a function of flux can be used as a useful way of constraining the cosmological model. The constraints can be dramatically improved if redshift information is provided for a small subsample of the observed clusters. In this work we present the constraints on $\Omega_{m}$ and $\sigma_{8}$ expected for a combined mm-optical survey. The mm survey (Planck) providing the cluster number counts as a function of flux and the optical survey (GTC) the redshift estimation of a small subsample of the cluster catalogue. We also show how it is possible to get an accurate estimate of the cluster flux using a non-parametric component separation algorithm and how using morphological redshifts it is possible to make a high-z preselection of the small subsample to be observed in the optical.
\end{abstract}

Keywords: Cosmology:theory - cosmological parameters.

\section{Constraining the cosmology with the SZE.}

Studies on the local abundance of clusters have shown that today's observed cluster density constrains the cosmological model. However, these constrains show a degeneracy between the matter density, $\Omega_{m}$, and the normalisation of the power spectrum, $\sigma_{8}$ (Bahcall \& Cen 1993). This degeneracy can be broken by including in the analysis the cluster abundance at high redshift. However, observations of clusters at high redshift are difficult because of the decrease of flux with redshift (flux $\propto D_{l}(z)^{-2}$ ) The peculiarity that the central decrement of the SZE is redshift independent, makes this effect an interesting way of observing high redshift clusters. Future mm experiments, like Planck, will detect thousands of clusters. A large proportion of these clusters will be at high redshift. Given such a large number of clusters, it is natural to think that the cluster catalogue provided by these experiments will be 
an important source of cosmological studies. However, since the SZE is redshift independent, these catalogues will contain no information about the redshift of the clusters. They will contain information only about the flux of the clusters. With the flux, it is possible to build the cluster number counts as a function of flux. By using this data set alone, one can not, for instance, distinguish between a $\Lambda$ CDM and an OCDM models both with the same matter content. Redshift information is essential to distinguish between these two models. However, we do not need to know the redshift of all the clusters in order to distinguish between these two models. An optically identified subsample of about 300 clusters (in the case of Planck) randomly selected from the SZE catalogue is enough to distinguish between the above models at a $3 \sigma$ level (see details in Diego et al. 2002a). By randomly we mean that from the SZE catalogue we pick up 300 clusters randomly selected from the full catalogue. In this random subsample there will be many low, intermediate and some high redshift clusters. We illustrate this situation in figure 1 where we compare the
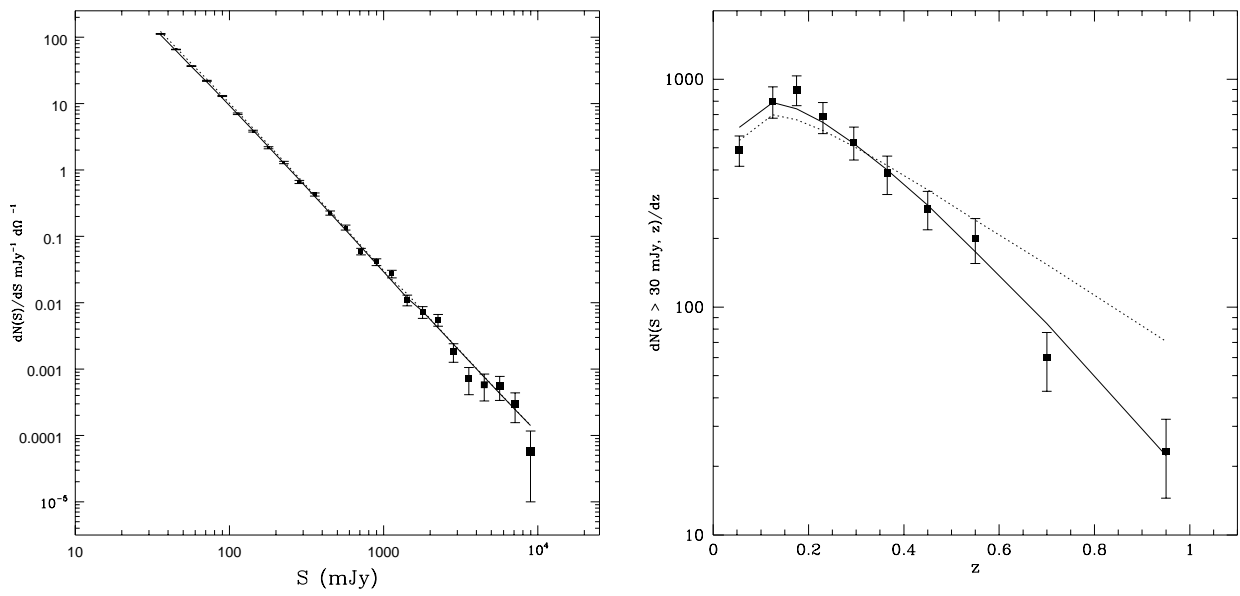

Figure 1. Taken from Diego et al. 2002a. Predicted cluster number counts for Planck (left) and number counts per redshift bin (right) for the GTC followup of the small subsample of 300 clusters. The lines show the predictions for the theoretical model. $\Lambda$ CDM solid line and OCDM dotted line $\left(\Omega_{m}=0.3, \sigma_{8}=0.8\right.$ in both cases).

above models with the expectations for the Planck satellite (left) and the optical followup of the subsample with 300 clusters performed by a 10-m class telescope like GTC. Using the data sets of figure 1 it is possible to constrain $\sigma_{8}$ and $\Omega_{m}$ within an accuracy of about 0.1 (Diego et al. 2002a). However, to get the data sets of figure 1 we need first to detect the clusters and estimate their flux and second to estimate the 
redshift of a small subsample $(\approx 300$ clusters $)$ of clusters. In the next two sections we present two techniques which will be useful to solve this problems.

\section{Cluster detection: Non-parametric method}

In this section we will give a brief description of the non-parametric method presented in Diego et al. 2002b which has demonstrated its robustness with simulated Planck data. The SZE produces a distortion in the $\mathrm{CMB}$ spectrum shifting the $\mathrm{CMB}$ photons to higher frequencies. This shift can be easily observed if we have multifrequency maps of the CMB. However multifrequency maps of the CMB also contain other components: Galactic, extragalactic and instrumental noise. The separation of all these components is the main aim of the different proposed component separation algorithms. Due to the large number of components involved in this problem, the component separation algorithms usually need to make many assumptions about the frequency dependence of each component and it angular power spectrum. These assumptions can produce a bias in the final result if some of the assumptions are wrong. There is still a lot of uncertainty about the frequency dependence of several components and/or about their angular power spectrum. The non-parametric method proposed in Diego et al. 2002b overcomes these problems by doing no assumptions except for the frequency dependence of the SZE (which is well known) and a weak assumption about the prior (however, the results seem to be almost insensitive to this assumption). In a few words, the non-parametric method deconvolves an optimally weighted combination of the multifrequency maps which have been previously pre-processed in order to increase the SNR of the SZE. When applied to Planck simulated data, the non-parametric method predicts that about 10000 clusters will be detected in all the sky. We show in figure 2 (left) how the non-parametric method is able to recover the fluxes which are our main interest to build the first of the data sets in figure 1 .

\section{Optical followup: Morphological redshifts}

Although in figure 1 we assumed that the subsample of 300 clusters was randomly selected from the SZE catalogue, the results would improve if the ratio of high-to-low redshift clusters is high. In order to achieve this high ratio it would be interesting to preselect the small subsample by redshift instead of performing a random preselection. But how to make this redshift preselection if the SZE is redshift independent ? In fact, although the spectrum of the SZE is redshift independent, there are observables like the total flux which do depend on the redshift 
of the cluster. By combining different SZE observables it is possible to get an estimate of the redshift (Diego et al. 2002c). Morphological redshifts can provide this estimate combining several observables in a principal component analysis (PCA). The principal components (pc) are then calibrated with a set of known redshifts and then a Bayesian estimator is used to get the most likely redshift of the clusters. In figure 2 (right) we show the performance of this technique applied using N-body high resolution simulations. These redshift estimates can then be used to preselect a subsample of high redshift cluster candidates or to optimise the followup of a randomly selected subsample by observing the low redshift candidates with small telescopes and the high redshift clusters with large telescopes.
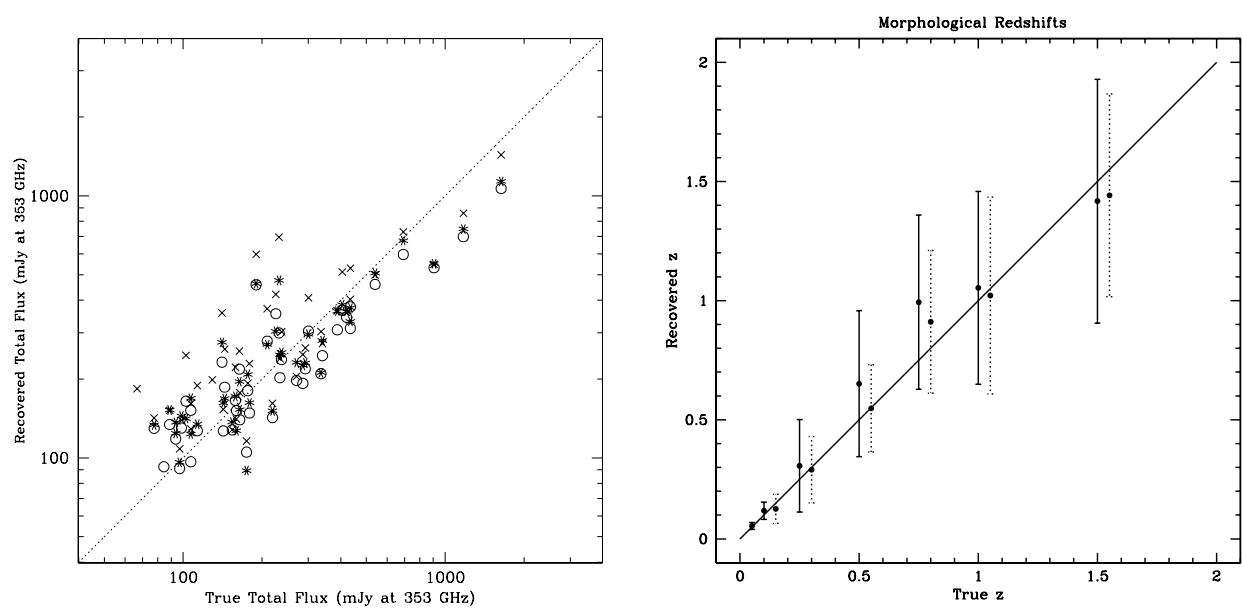

Figure 2. Left. Taken from Diego et al. 2002b. Stability on the recovered fluxes. The different symbols correspond to different assumptions on the prior. The result shows to be quite insensitive to this assumption demonstrating the robustness of the method. Right. Taken from Diego et al. 2002c. Performance of the morphological redshifts for a survey with $\mathrm{FWHM}=25$ arcsec resolution. The method improves if only the first pc is used in the high redshift interval (dotted errors). However, at low redshifts, the other pcs help to reduce the error in the $z$-estimation (solid errors).

\section{References}

Bahcall N.A, Cen R. 1993, ApJ, 407, L49.

Diego J.M., Martínez-González E., Sanz J.L., Benitez N., Silk J. 2002a, MNRAS, 331, 556.

Diego J.M., Vielva P., Martínez-González E., Silk J., Sanz J.L. 2002b, MNRAS, 336, 1351.

Diego J.M., Mohr J, Silk J, Bryan G. MNRAS submitted. Preprint astro-ph/0207353. 